\title{
亶 \\ Malformação congênita em gambá-de-orelha-branca (Didelphis albiventris Lund 1840)
}

[Congenital malformation in white-eared opossum (Didelphis albiventris Lund 1840)]

"Relato de Caso/Case Report"

Caio Henrique Oliveira Carniatto*, Jussara Maria Leite Oliveira Leonardo

Departamento de Medicina Veterinária do Centro Universitário Cesumar - UniCesumar, Maringá-PR, Brasil.

*Autor para correspondência/Corresponding author: E-mail: caiocarniatto@gmail.com

\section{Resumo}

A medicina zoológica estuda os animais silvestres, nativos ou não, de vida livre ou mantidos em zoológicos. Embora o gambá-de-orelha-branca (Didelphis albiventris) seja um marsupial abundante no Brasil, não são relatados casos de malformação congênita na espécie. Considerando a necessidade de se conhecer os aspectos clínicos e patológicos em animais silvestres, relatou-se um caso de malformação congênita em um juvenil de gambá-de-orelha-branca. O animal foi encaminhado para o Hospital Veterinário da UniCesumar (Maringá/Paraná), após ser encontrado em um fragmento florestal no município. Na anamnese, constatou-se que o paciente era dócil, debilitado, não se alimentava sozinho e sua dieta era composta por carne bovina e frango, peixes, ovos cozidos, maçã e banana. No exame clínico verificou-se que o animal apresentava mucosas normocoradas, hidratação regular e linfonodos não reativos. A língua era permanentemente mantida exposta, os caninos maxilares eram aparentes e a cauda apresentava desvios ósseos nos sentidos dorsoventral e laterolateral. Em exame radiográfico, constatou-se que o paciente apresentava desvio da fíbula esquerda, desvios ósseos na cauda e no corpo da mandíbula. O paciente foi mantido por um tutor em residência, visando manter o animal estável e nutrido. Dois meses após a primeira consulta, o tutor entrou em contato para comunicar que o animal havia morrido. Malformações congênitas são comuns em diferentes espécies, mas sua descrição em animais de vida livre é rara e esporádica, dificultando traçar dados epidemiológicos e fatores etiológicos. Estudos devem ser conduzidos visando quantificar casos de malformações em espécies silvestres, as preservando na natureza.

Palavras-chave: animais silvestres; anomalias congênitas; gambá-de-orelha-branca; patologia.

\begin{abstract}
Zoological medicine studies the wildlife, native or not, free-living or kept in zoos. Although the white-eared opossum (Didelphis albiventris) is an abundant marsupial in Brazil, no cases of congenital malformation in the species are reported. Considering the need to know the clinical and pathological aspects in wildlife, a case of congenital malformation in a $D$. albiventris was reported. The animal was sent to the Veterinary Hospital of UniCesumar (Maringá/Paraná), after being found in a forest fragment. In the anamnesis, the patient was docile, fragile and did not eat alone, and his diet consisted of meats, fish, boiled eggs and various fruits. On clinical examination, it was found that the animal had normal colored mucous membranes, regular hydration and non-reactive lymph nodes. The tongue was kept permanently exposed, the maxillary canines were apparent and the tail presented bone deviations in the dorsoventral and laterolateral directions. On radiographic examination, the patient was found to have deviation of the left fibula, bone deviations in the tail and body of the mandible. The patient was fostered in a residence, aiming to keep the animal stable and nourished. Two months after the first consultation, the person responsible for the animal reported that the animal had died. Congenital malformations are common in different species, but description in free-living animals is rare and sporadic, making it difficult to trace epidemiological data and etiological factors. Studies should be conducted to quantify cases of malformations in wild species, in order to preserve the species in nature.
\end{abstract}

Keywords: congenital anomalies; opossum; pathology; veterinary medicine; wild animals 


\section{Introdução}

O gambá-de-orelha-branca (Didelphis albiventris) é uma espécie solitária, noturna e terrestre (Abdala et al., 2001; Almeida et al., 2008; Schimming et al., 2016), amplamente distribuída na América do Sul, ocupando uma série de habitats que vão desde o cerrado até florestas (Lemos e Cerqueira, 2002; Teixeira et al., 2006; Cáceres et al., 2010; Nascimento et al., 2018), incluindo áreas urbanas (Cantor et al., 2010). Em cidades, esta espécie atua em processos de reflorestamento, dispersando sementes de frutos consumidos. Assim como outras espécies do gênero Didelphis, o gambá-de-orelha-branca é onívoro e sua alimentação varia de acordo com a disponibilidade de alimento (Aguiar et al., 2004; Silva et al., 2014).

O gambá-de-orelha-branca (D. albiventris) pertence à Infraclasse Marsupialia e ordem Didelphimorphia (Nilsson et al., 2004), cujas fêmeas da espécie apresentam marsúpio (Bertassoli et al., 2013), períodos gestacionais curtos (Samoto et al., 2006) e útero duplo (Gonçalves et al., 2009), e os machos possuem glande bífida e com pontas delgadas (Matheus et al., 2011).

Embora a espécie seja amplamente estudada em seus aspectos morfológicos (Bertassoli et al., 2015; Inamassu et al., 2017; Massari et al., 2019), ecológicos (Cáceres et al., 2016; Junges et al., 2017; Sá et al., 2018) e sanitários (Humberg et al., 2012; Zabott et al., 2017; Massini et al., 2019), não há relatos de malformações congênitas.

Malformações congênitas são defeitos que afetam a anatomia estrutural e funcional de órgãos internos (Caldas et al., 2014), podendo gerar cardiopatias (Oliveira et al., 2018), disfunção respiratória (Andrade et al., 2011), fissura craniofacial (Lopes et al., 2019), malformação vertebral cervical (Fonteque et al., 2018) e anomalias uterinas (Bessa et al., 2020), e ocorrem nas fases embrionárias e fetais do desenvolvimento (Dantas et al., 2010).

A etiologia das malformações é diversa e nem sempre detectável (Nicácio et al., 2013), sendo um desafio na prática veterinária (MarcolongoPereira et al., 2010). Podem ter origem genética e ambiental (Sant'Anna et al., 2013), incluindo ingestão de plantas tóxicas (Marcelino et al., 2017), consumo de agrotóxicos (Dutra e Ferreira, 2017), infecções (Tarlinton et al., 2012; Agerholm et al., 2015; Santos et al., 2016) e intoxicação por metais pesados (Govind e Madhuri, 2014) e compostos químicos (Ayari et al., 2017).
Visando contribuir para o conhecimento patológico e epidemiológico em animais silvestres, este estudo teve como objetivo relatar um caso de malformação congênita em um juvenil de gambáde-orelha-branca (D. albiventris) em Maringá/Paraná, Sul do Brasil.

\section{Descrição do Caso}

Em 24 de fevereiro de 2017, foi atendido no Hospital Veterinário da UniCesumar (Maringá/PR), um gambá-de-orelha-branca ( $D$. albiventris), com idade estimada de seis meses, macho e pesando $0,258 \mathrm{~kg}$, mantido por um tutor em residência. $O$ animal, quando filhote, foi encontrado sozinho em um fragmento florestal após dias de chuva, em estado de choque. O paciente foi mantido sob tutoria por cinco meses, antes da primeira consulta.

$\mathrm{O}$ tutor encaminhou o paciente para atendimento veterinário por se tratar de um animal silvestre, procurando identificar possíveis doenças infectocontagiosas e orientações para manter o animal em cativeiro.

$\mathrm{Na}$ anamnese o tutor relatou que o animal era dócil, debilitado, não se alimentava sozinho e sua dieta era composta por carne bovina e frango, peixes, ovos cozidos, maçã e banana. No exame clínico verificou-se que o animal apresentava mucosas normocoradas, hidratação regular e linfonodos não reativos. A língua era permanentemente mantida exposta (Figura 1A). Notou-se que os caninos maxilares eram aparentes (Figura 1B), e a cauda apresentava desvios ósseos nos sentidos dorsoventral e laterolateral (Figura 1C).

Solicitou-se um exame radiográfico, no qual foi constatado que o paciente apresentava desvio da fíbula esquerda (Figura 2A), desvios ósseos na cauda (Figura 2B) e desvio ósseo no corpo da mandíbula (Figura 2C).

Não foi prescrito medicamento durante o tratamento veterinário e, dois meses após a primeira consulta, o tutor entrou em contato para comunicar que o animal havia morrido. O corpo, porém, não foi encaminhado para necropsia.

\section{Discussão}

$\mathrm{O}$ presente relato reportou um caso de malformação congênita em um gambá-de-orelhabranca (Didelphis albiventris), juvenil e de vida livre. Malformações congênitas são resultado de mutações genéticas ou cromossômicas (Albarella et al., 2017), podendo ser hereditárias (Gholap et 
al., 2014). Nesse contexto, alterações genéticas são consideradas fatores etiológicos de mutações e consequentemente malformações em mamíferos (Ghebranious et al., 2008), e podem afetar apenas determinadas áreas do corpo (Souza et al., 2018; Araujo et al., 2019) ou todo o organismo (Booth et al., 2013), sendo, dessa forma, incompatível com a vida.

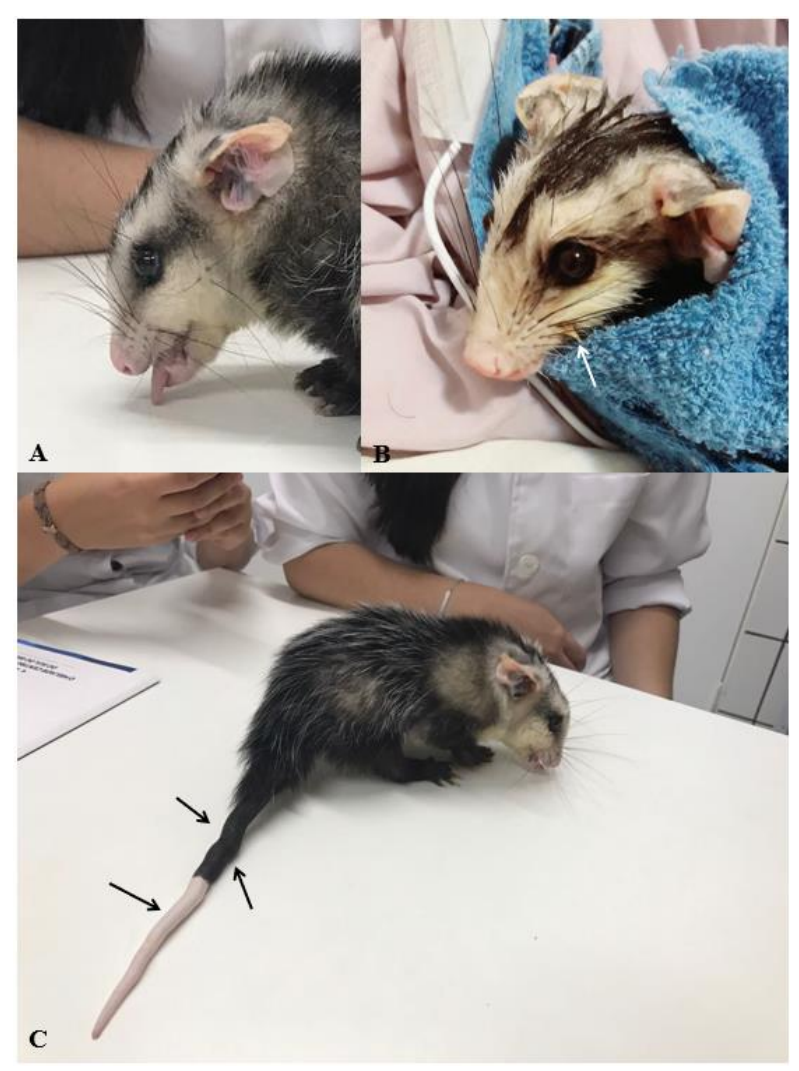

Figura 1. Anamnese de gambá-de-orelha-branca (Didelphis albiventris) com malformação congênita. A) Notar a língua permanentemente exposta. B) $\mathrm{O}$ canino maxilar aparente, mesmo com o animal de boca fechada (seta). C) Notar os desvios ósseos na cauda (setas).

Malformações também podem ter origem ambiental (Pourlis, 2011), como as decorrentes de ações antrópicas, que vem causando efeitos potencialmente irreversíveis e afetam a sanidade de animais silvestres, principalmente os que convivem intimamente em áreas florestais dentro de grandes cidades (Tompkins et al., 2011; Ruoppolo e Robinson, 2014; Hoy et al., 2015; Plaza e Lambertucci, 2017). Ferraz et al. (2014) relataram que o estresse pode ocasionar malformação fetal em primatas cativos, causando a morte dos fetos.

Além da interferência humana, deve-se considerar outras causas de malformações congênitas em mamíferos, como cruzamento de animais consanguíneos (Agerholm et al., 2001; Scaglione et al., 2010) e deficiências em vitamina A (Baker e Lyon, 1977; Giadinis et al., 2012).

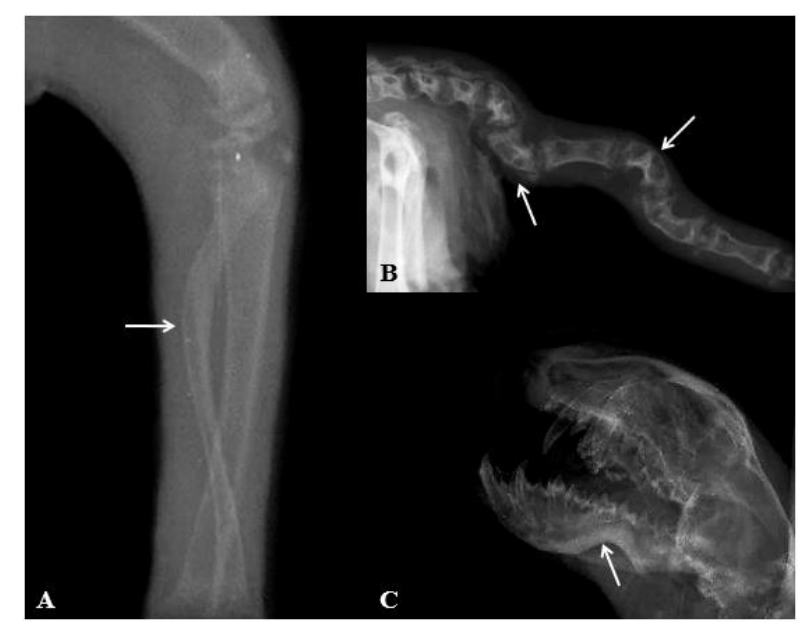

Figura 2. Radiografia de gambá-de-orelha-branca (Didelphis albiventris) com malformação congênita. A) Desvio ósseo da fíbula esquerda (seta). B) Desvios ósseos das vértebras caudais (seta). C) Desvio ósseo no corpo da mandíbula (seta).

Embora malformações em mamíferos sejam esporadicamente relatadas, dificultando compreender qual sua incidência e prevalência em populações silvestres, alterações estruturais em anfíbios indicam que malformações podem estar diretamente relacionadas à poluição e contaminações por pesticidas, radiação e fertilizantes (Taylor et al., 2005; Reeves et al., 2008; Koleska e Jablonski, 2016). Na espécie relatada são reportados molares supranumerários (Astúa de Moraes et al., 2001) e anomalias dentárias (Chemisquy e Martin, 2016).

Diversas pesquisas têm demonstrado o comprometimento anatomofisiológico de malformações congênitas em animais domésticos como gatos (Martini-Santos et al., 2012; Miranda et al., 2013), cães (Lopes et al., 2019), equinos (Rocha et al., 2007), bovinos (Pavarini et al., 2008; Macêdo et al., 2011), cordeiros (Nóbrega Jr et al., 2005; Santos et al., 2012) e cabras (Medeiros et al., 2005), além de animais silvestres como golfinhos (Berghan e Visser, 2000; Powell et al., 2009; Laeta et al., 2010) e leões (Scaglione et al., 2010; Saragusty et al., 2014).

$\mathrm{O}$ animal relatado neste estudo apresentou malformações externas, observadas na anamnese, que comprometiam os sistemas ósseo e muscular, dificultando processos básicos como a deglutição. No presente relato não foi possível identificar alterações estruturais e funcionais em órgãos 
internos, pois o animal não foi encaminhado para necropsia.

Este é o primeiro relato de malformação congênita em $D$. albiventris. Demais estudos devem ser conduzidos visando descrever e quantificar malformações em populações silvestres, bem como seus fatores etiológicos, contribuindo para a conservação das espécies na natureza.

\section{Conflito de interesse}

Os autores declaram não existir conflito de interesse.

\section{Referências}

Abdala, F.; Flores, D.A.; Giannini, N.P. Postweaning ontogeny of the skull of Didelphis albiventris. Journal of Mammalogy, 82(1): 190-200, 2001.

Agerholm, J.S.; Bendixen, C.; Andersen, O.; Arnbjerg, J. Complex vertebral malformation in Holstein calves. Journal of Veterinary Diagnostic Investigation, 13: 283-289, 2001.

Agerholm, J.S.; Hewicker-Trautwein, M.; Windsor, P.A. Virus-induced congenital malformations in cattle. Acta Veterinaria Scandinavica, 57(54): 1-14, 2015.

Aguiar, M.S.; Ferigolo, J.; Rossi Junior, J.L.; Gioso, M.A. Atrição dental em Didelphis albiventris e D. marsupialis (Marsupialia, Didelphimorphia, Didelphidae) do Sul do Brasil. Ciência Rural, 34(4): 1127-1132, 2004. Albarella, S.; Ciotola, F.; D’Anza, E.; Coletta, A.; Zicarelli, L.; Peretti, V. Congenital malformations in river buffalo (Bubalus bubalis). Animals, 7(2): 1-15, 2017.

Almeida, A.; Torquetti, C.G.; Talamoni, S.A. Use of space by neotropical marsupial Didelphis albiventris (Didelphimorphia: Didelphidae) in an urban forest fragment. Revista Brasileira de Zoologia, 25(2): 214-219, 2008.

Andrade, C.F.; Ferreira, H.P.C.; Fisher, G.B. Malformações pulmonares congênitas. Jornal Brasileiro de Pneumologia, 37(2): 259-271, 2011.

Araujo, H.B.; Costa-Pinto, D.; Castro, C.S.C.; Pires, J.R. A case of polymelia associated with syndactyly in Didelphis aurita (Wied-Neuwied, 1826). Brazilian Journal of Biology, In Press, 1-5, 2019.

Astúa de Moraes, D.A; Lemos, B.; Cerqueira, R. Supernumerary molars in neotropical opossums
(Didelphimorphia, Didelphidae). Mammalian Biology, 66: 193-203, 2001.

Ayari, T.E.; Mleiki, A.; Menif, N.T.E. Genitalia malformations in Stramonita haemastoma (Gastropoda: Muricidae) from Atlantic and Mediterranean Coast. Journal of Aquaculture Research \& Development, 8(6): 1-5, 2017.

Baker, J.R.; Lyon, D.G. Skull malformation and cerebellar herniation in captive African lions. Veterinary Record, 100(8): 154-156, 1977.

Berghan, J.; Visser, I.N. Vertebral column malformations in New Zealand delphinids with a review of cases worldwide. Aquatic Mammals, 26(1): 17-25, 2000.

Bertassoli, B.M.; Santos, A.C.; Oliveira, F.D.; Oliveira, D.M.; Assis-Neto, A.C.; Carvalho, A.F. Morfologia da laringe e traqueia de gambás (Didelphis sp.). Ciência Animal Brasileira, 14(2): 222-229, 2013.

Bertassoli, B.M.; Santos, A.C.; Lessa, T.B.; Santos, I.G.D.; Copola, A.G.L.; Mançanares, C.A.F.; Silva, G.A.B.; Jorge, E.C. Morphological characteristics of the neonate of the white-eared opossum (Didelphis albiventris Lund, 1840) (Metatheria: Didelphidae). Brazilian Journal of Biological Sciences, 2(4): 209-219, 2015.

Bessa, V.P.; Silva e Silva, P.; Viana Junior, M. F.; Monteiro, T.V.R.; Silva, V.A.S.; Carvalho, A.H.G.G. Malformações congênitas em cadela: Útero unicórnio e hipoplasia renal - relato de caso. Brazilian Journal of Animal and Environmental Research, 3(3): 2488-2492, 2020.

Booth, J.L.; Peng, X.; Baccon, J.; Cooper, T.K. Multiple complex congenital malformations in a rabbit kit (Oryctolagus cuniculi). Comparative Medicine, 63(4): 342-347, 2013.

Cáceres, N.C.; Graipel, M.E.; Monteiro-Filho, E.L.A. Técnicas de observação e amostragem de marsupiais. In: Reis, N.R.; Peracchi, A.L.; Rossaneis, B.K.; Fregonezi, M.N. Técnicas de estudos aplicadas aos mamíferos silvestres brasileiros. $1^{\text {a }}$ ed. Rio de Janeiro: Technical Books, 2010. p. 21-36.

Cáceres, N.C.; Weber, M.M.; Melo, G.L.; Meloro, C.; Sponchiado, J.; Carvalho, R.S.; Bubadué, J.M. Which factors determine spatial segregation in the South American opossums (Didelphis aurita and D. albiventris)? An ecological niche modelling and geometric morphometrics approach. PLoS ONE, 11(6): 119, 2016. 
Caldas, S.A.; Nogueira, V.A.; Lima, A.E.S.; Aragão, A.P.; d'Avila, M.S.; Santos, A.M.; Miranda, I.C.; Costa, S.Z.R.; Peixoto, T.C. Queilosquise bilateral em bovino - Relato de caso. Revista Brasileira de Medicina Veterinária, 36(1): 55-59, 2014.

Cantor, M.; Ferreira, L.A.; Silva, W.R.; Setz, E.Z.F. Potential seed dispersal by Didelphis albiventris (Marsupialia, Didelphidae) in highly disturbed environment. Biota Neotropica, 10(2): 45-51, 2010.

Chemisquy, M.A.; Martin, G.M. Dental anomalies in Didelphis albiventris (Mammalia, Marsupialia, Didelphidae) from Argentina, Brazil and Uruguay. Iheringia. Série Zoologia, 106: 1-10, 2016.

Dantas, A.F.M.; Riet-Correa, F.; Medeiros, R.M.T.; Galiza, G.J.N.; Pimentel, L.A.; Anjos, B.L.; Mota, R.A. Malformações congênitas em ruminantes no semiárido do Nordeste Brasileiro. Pesquisa Veterinária Brasileira, 30(10): 807-815, 2010.

Dutra, L.S.; Ferreira, A.P. Associação entre malformações congênitas e a utilização de agrotóxicos em monoculturas no Paraná, Brasil. Saúde e Debate, 41(2): 241-253, 2017.

Fonteque, J.H.; Souza, A.F.; Muller, T.R.; Gonçalves, R.P.M.; Granella, M.C.S.; Mendes, R.P.; Schade, J. Malformação vertebral cervical congênita em cordeiros. Acta Scientiae Veterinariae, 46(1): 1-5, 2018.

Ghebranious, N.; Blank, R.D.; Raggio, C.L.; Staubli, J.; McPherson, E.; Ivacic, L.; Rasmussen, K.; Jacobsen, F.S.; Faciszewski, T.; Burmester, J.K.; Pauli, R.M.; Boachie-Adjei, O.; Glurich, I.; Giampietro, P.F. A Missense $T$ (Brachyury) mutation contributes to vertebral malformations. Journal of Bone and Mineral Research, 23(10): 1576-1583, 2008.

Gholap, P.N.; Kale, D.S.; Sirothia, A.R. Genetic diseases in cattle: A review. Research Journal of Animal, Veterinary and Fishery Sciences, 2(2): 24-33, 2014.

Giadinis, N.D.; Papaioannou, N.G.; Tsaousi, P.; Koutsoumpas, A.; Fytianou, A.; Karatzias, H. Vertebral absence in a lamb with Vitamin A deficiency. Pakistan Veterinary Journal, 32(2): 295-297, 2012.

Gonçalves, N.N.; Maçanares, C.A.F.; Miglino, M.A.; Samoto, V.Y.; Martins. D.S.; Ambrósio, C.E.; Ferraz, R.H.S.; Carvalho, A.F. Aspectos morfológicos dos órgãos genitaisfemininos do gambá (Didelphis sp.). Brazilian Journal of
Veterinary Research and Animal Science, 46(4): 332-338, 2009.

Govind, P.; Madhuri, S. Heavy metals causing toxicity in animals and fisches. Research Journal of Animal, Veterinary and Fishery Sciences, 2(2): 17-23, 2014.

Hoy, J.; Swanson, N.; Seneff, S. The high cost of pesticides: Human and animal diseases. Poultry, Fiseheries and Wildlife Sciences, 3(1): 1-18, 2015.

Humberg, R.M.P.; Oshiro, E.T.; Pires e Cruz, M.S.; Ribolla, P.E.M.; Alonso, D.P.; Ferreira, A.M.T.; Bonamigo, R.A.; Tasso Jr, N.; Oliveira, A.G. Short report: Leishmania chagasi in opossums (Didelphis albiventris) in an urban area endemic for visceral leishmaniasis, Campo Grande, Mato Grosso do Sul, Brazil. American Journal of Tropical Medicine and Hygiene, 87(3): 470-472, 2012.

Inamassu, L.R.; Mamprim, M.J.; Dadalto, C.R.; Cavaletti, F.C.; Mello, M.C.; Schimming, B.C. Absence of bony patella in the white-eared opossum (Didelphis albiventris): Morphology and diagnostic imaging. Anatomia, Histologia, Embryologia, 46(6): 611-614, 2017.

Junges, S.O.; Consatti, G.; Périco, E.; Bordignon, S.A.L.; Freitas, E.M.; Cademartori, C.V. Endozoochory by Didelphis albiventris Lund, 1840 (Mammalia, Didelphimorphia) in a Semideciduous Seasonal Forest remnant in the South of Brazil. Biota Neotropica, 18(1): 1-8, 2017.

Koleska, D.; Jablonski, D. Two cases of unclear hindlimb malformation in Bombina variegata. Ecologica Montenegrina, 9: 56-58, 2016.

Laeta, M.; Souza, S.M.F.M.; Siciliano, S. Anomalias ósseas congênitas em Sotalia guianensis (Mammalia, Cetacea, Delphinidae) da costa centro-norte do Estado do Rio de Janeiro. Pesquisa Veterinária Brasileira, 30(6): 484-490, 2010.

Lemos, B.; Cerqueira, R. Morphological differentiation in the white-eared opossum group (Didelphidae: Didelphis). Journal of Mammalogy, 83(2): 354-369, 2002.

Lopes, T.H.L.; Barros, H.B.S.; Freitas, J.C.C.; Domingos, L.S.; Araújo, A.F.; Figueiredo, M.A.P.; Schons, S.V.; Manrique, W.G. Malformações faciais congênitas em cães: Relato de caso. Ars Veterinaria, 35(2): 73-77, 2019.

Macêdo, J.T.S.A.; Lucena, R.B.; Giaretta, P.R.; Kommers, G.D.; Fighera, R.A.; Irigoyen, L.F.; 
Barros, C.S.L. Defeitos congênitos em bovinos da Região Central do Rio Grande do Sul. Pesquisa Veterinária Brasileira, 31(4): 297306, 2011.

Marcelino, S.A.C.; Macêdo, J.T.S.A.; Reis, S.D.S.; Lacerda, M.S.C.; Silva, A.R.S.; Riet-Correa, F.; Pimentel, L.A.; Pedroso, P.M.O. Malformações em pequenos ruminantes no semiárido da Bahia: Aspectos epidemiológicos, clínicopatológicos e radiológicos. Pesquisa Veterinária Brasileira, 37(12): 1437-1442, 2017.

Marcolongo-Pereira, C.; Schild, A.L.; Soares, M.P.; Vargas Jr, S.F.; Riet-Correa, F. Defeitos congênitos diagnosticados em ruminantes na Região Sul do Rio Grande do Sul. Pesquisa Veterinária Brasileira, 30(10), 816-826, 2010. Martini-Santos, B.J.; Diefenbach, A.; Peixoto, T.C.; Nóbrega Jr, J. E.; Anjos, B.L. Anomalias congênitas múltiplas em felinos domésticos neonatos. Acta Scientiae Veterinariae, 40(4): 1-6, 2012.

Massari, C.H.A.L.; Pinto, A.C.B.C.F.; Carvalho, Y.K.; Silva, A.F.; Miglino, M.A. Volumetric computed tomography reconstruction, rapid prototyping and 3D printing of opossum head (Didelphis albiventris). International Journal of Morphology, 37(3): 838-844, 2019.

Massini, P.F.; Drozino, R.N.; Otomura, F.H.; Mongruel, A.C.B.; Valente, J.D.M.; Toledo, M.J.O.; Martins, T.F.; Vidotto, O.; Vieira, T.S.W.J.; Vieira, R.F.C. Detection of hemotropic Mycoplasma sp. in white-eared opossums (Didelphis albiventris) from Southern Brazil. Brazilian Journal of Veterinary Parasitology, 28(4): 797-801, 2019.

Matheus, S.M.M.; Guazzelli Filho, J.; Pinto e Silva, J.R.C.; Cesário, M.D. Aspectos morfológicos do pênis do gambá Sul Americano (Didelphis albiventris). Revista Científica Eletrônica de Medicina Veterinária, 9(17): 1-12, 2011.

Miranda, D.F.H.; Azevedo Júnior, J.W.C.; Ferraz, M.S.; Pessoa, G.T.; Bezerra, D.O.; Fortes, E.A.M.; Conde Júnior, A.M. Malformações congênitas em neonatos felinos: Relato de caso. PUBVET, 7(4): 1-8, 2013.

Medeiros, J.M.; Tabosa, I.M.; Simões, S.V.D.; Nóbrega Jr, J.E.; Vasconcelos, J.S.; RietCorrea, F. Mortalidade perinatal em cabritos no semi-árido da Paraíba. Pesquisa Veterinária Brasileira, 25(4): 201-206, 2005.
Nascimento, D.C.; Campos, B.A.T.P.; Fraga, E.C.; Barros, M.C. Genetic variability of populations of the white-eared opossum, Didelphis albiventris Lund 1840 (Didelphimorphia; Didelphidae) in Brazil. Brazilian Journal of Biology, 79(4): 1-9, 2018.

Nicácio, M.A.C.; Carvalho, G.D.; Serrano, M.T.L.; Lang, A.; Santana, M. L. Espinha bífida aberta em bovino: Primeiro relato no estado de Minas Gerais. Arquivo Brasileiro de Medicina Veterinária e Zootecnia, 65(2): 341-345, 2013.

Nilsson, M.A.; Arnason, U.; Spencer, P.B.S.; Janke, A. Marsupial relationships and a timeline for marsupial radiationin South Gondwana. Gene, 340: 189-196, 2004.

Nóbrega Jr, J.E.; Riet-Correa, F.; Nóbrega, R.S.; Medeiros, J.M.; Vasconcelos, J.S.; Simões, S.V.D.; Tabosa, I M. Mortalidade perinatal de cordeiros no semi-árido da Paraíba. Pesquisa Veterinária Brasileira, 25(3), 171-17, 2005.

Oliveira, P.H.A.; Souza, B.S.; Pacheco, E.N.; Menegazzo, M.S.; Corrêa, I.S.; Zen, P.R.G.; Rosa, R.F.M.; Cesa, C.C.; Pellanda, L.C.; Vilela, M.A.P. Síndromes genéticas associadas a defeitos cardíacos congênitos e alterações oftalmológicas - Sistematização para o diagnóstico na pratica clínica. Arquivos Brasileiros de Cardiologia, 110(1): 84-90, 2018.

Pavarini, S.P., Sonne, L.; Antoniassi, N.A.B.; Santos, A.S.O.; Pescador, C.A.; Corbellini, L.G.; Driemeier, D. Anomalias congênitas em fetos bovinos abortados no Sul do Brasil. Pesquisa Veterinária Brasileira, 28(3), 149154, 2008.

Plaza, P.I.; Lambertucci, S.A. How are garbage dumps impacting vertebrate demography, health, and conservation? Global Ecology and Conservation, 12: 9-20, 2017.

Pourlis, A.F. Developmental malformations in Avian species. Manifestations of unknown or genetic etiology - a review. Asian Journal of Animal and Veterinary Advances, 6 (5): 401415, 2011.

Powell, J.W.B.; Archibald, R.T.; Cross, C.A.; Rotstein, D.S.; Soop, V.M.; McFee, W.E. Multiple congenital cardiac abnormalities in an atlantic bottlenose dolphin (Tursiops truncatus). Journal of Wildlife Diseases, 45(3): 839-842, 2009.

Reeves, M.K.; Dolph, C.L.; Zimmer, H.; Tjeerdema, R.S.; Trust, K.A. Road proximity 
increases risk of skeletal abnormalities in wood frogs from National Wildlife Refuges in Alaska. Environmental Health Perspectives, 116(8): 1009-1014, 2008.

Rocha, T.G.; Teixeira, L.G.; Seppa, G.S.; França, T.N.; Brito, M.F. Fístulas e outras anomalias congênitas dos tratos digestivo e urinário em um potro. Ciência Rural, 37(5): 1488-1491, 2007.

Ruoppolo, V.; Robinson, I. Emergências ambientais - Ações para a redução dos impactos à fauna. In: Cubas, Z.S.; Silva, J.C.R.; CatãoDias, J.L. Tratado de animais selvagens: Medicina veterinária. 2 ed. São Paulo: Roca, 2014. p. 2327-2338.

Sá, E.F.G.G.; Rodrigues, V.S.; Garcia, M.V.; Zimmermann, N. P.; Ramos, V.N.; Blecha, I.M.Z.; Duarte, P.O.; Martins, T.F.; Bordignon, M.O.; Andreotti, R. Ticks on Didelphis albiventris from a Cerrado area in the Midwestern Brazil. Systematic \& Applied Acarology, 23(5): 935-945, 2018.

Samoto, V.Y.; Miglino, M.A.; Ambrósio. C.E.; Pereira, F.T.V.; Lima, M.C.; Carvalho, A.F. Morfologia da glândula mamária de gambás da espécie Didelphis sp associada ao modelo marsupial. Biota Neotropica, 6(2): 1-12, 2006.

Sant'Anna, S.S.; Grego, K.F.; Lorigados, C.A.B.; Fonseca-Pinto, A.C.B.C.; Fernandes, W.; SáRocha, L.C.; Catão-Dias, J.L. Malformations in neotropical viperids: Qualitative and quantitative analysis. Journal of Comparative Pathology, 149(4): 503-508, 2013.

Santos, J.R.S.; Dantas, A.F.M., Riet-Correa, F. Malformações, abortos e mortalidade embrionária em ovinos causada pela ingestão de Mimosa tenuiflora (Leguminosae). Pesquisa Veterinária Brasileira, 32(11): 1103-1106, 2012.

Santos, T.R.; Faria, G.S.M.; Guerreiro, B.M.; Pietro, N.H.P.S.; Lopes, W.D.Z.; Silva, H.M.; Garcia, J.L.; Luvizotto, M.C.R.; Bresciani, K.D.S.; Costa, A.J. Congenital toxoplasmosis in chronically infected and subsequently challenged ewes. PLoS ONE, 11(10): 1-17, 2016.

Saragusty, J.; Shavit-Meyrav, A.; Yamaguchi, N.; Nadler, R.; Bdolah-Abram-T.; Gibeon, L.; Hildebrandt, T.B.; Shamir, M.H. Comparative skull analysis suggests speciesspecificcaptivity-related malformation in lions (Panthera leo). PLoS ONE, 9(4): 1-7, 2014.

Scaglione, F.E.; Schröder, C.; Degiorgi, G.; Zeira, O.; Bollo, E. Cranial malformations in related white lions (Panthera leo krugeri). Veterinary Pathology, 47(6): 1095-1099, 2010.

Schimming, B.C.; Reiter, L.F.F.; Saldoval, L.M.; Filadelpho, A.L.; Inamassu, L.R.; Mamprim, M. J. Anatomical and radiographic study of the white-eared opossum (Didelphis albiventris) skull. Pesquisa Veterinária Brasileira, 36(11): 1132-1138, 2016.

Silva, A.R.; Forneck, E.D.; Bordignon, S.A.L.; Cademartori, C.V. Diet of Didelphis albiventris Lund, 1840 (Didelphimorphia, Didelphidae) in two periurban areas in southern Brazil. Acta Scientiarum. Biological Sciences, 36(2): 241247, 2014.

Souza, A.J.S.; Silva, C.A.M.; Soares, M.C.P.; Cogliati, B.; Sá, L.R.M. Focal nodular hyperplasia of the liver in a free-ranging opossum (Didelphis marsupialis). Acta Scientiae Veterinariae, 46(1): 1-6, 2018.

Tarlinton, R.; Daly, J.; Dunham, S.; Kydd, J. The challenge of Schmallenberg virus emergence in Europe. The Veterinary Journal, 194(1): 1018, 2012.

Taylor, B.; Skelly, D.; Demarchis, L.K.; Slade, M.D.; Galusha, D.; Rabinowitz, P.M. Proximity to pollution sources and risk of amphibian limb malformation. Environmental Health Perspectives, 113(11): 1497-1501, 2005.

Teixeira, C.P.; Hirsch, A.; Perini, H.; Young, R.J. Marsupials from space: Fluctuating asymmetry, geographical information systems and animal conservation. Proceedings of the Royal Society B: Biological Sciences, 273(1589): 1007-1012, 2006.

Tompkins, D.M.; Dunn, A.M.; Smith, M.J.; Telfer, S. Wildlife diseases: From individuals to ecosystems. Journal of Animal Ecology, 80: 19-38, 2011.

Zabott, M.V.; Pinto, S.B.; Viott, Gruchouskei, L.; Bittencourt, L.H.F.B. Helmintofauna de Didelphis albiventris (Lund, 1841) no município de Palotina, Paraná, Brasil. Arquivos de Ciências Veterinárias e Zoologia da UNIPAR, 20(1): 19-22, 2017. 